\title{
Surgical cover for angioplasty
}

The need for on-site surgical cover for coronary angioplasty provokes discussion throughout the world and we thought that it would be valuable to obtain an international perspective on this subject. We approached five cardiologists in different countries, each performing a large number of coronary angioplasties. We asked them for some basic statistics, both national and institutional, on coronary angioplasty in 1992 so that readers can see the working circumstances on which these opinions are based.

When PTCA was first introduced it was appropriate to take precautions and provide surgical stand-by with what was then the standard form of interventional treatment. Is this approach still valuable or even relevant? With the advent of stents and perfusion balloons can't interventionists bail themselves out? We asked our commentators to consider the following issues:

- Is PTCA without on-site cardiac surgery acceptable? If not, why not? If you believe it is, what issues do you discuss with the patients before the procedure and what morbidity and mortality do you regard as acceptable?

- Has PTCA without surgical cover become more acceptable since the advent of stents and perfusion catheters?

- What is the risk of dissection of the left main vessel during coronary intervention? Can it be dealt with by non-surgical means?

- Does your country have national guidelines on on-site surgical stand-by? Does your personal policy differ from the national policy?

- Is there a need for surgical stand-by when an intervention is carried out on an infarct related artery? Are there other clinical circumstances that you would regard as a low risk PTCA?

- If you believe that on-site cardiac surgical cover is necessary for PTCA, do you believe that there is a similar need to cover diagnostic coronary angiography?

There are of course other relevant factors such as training, centralisation of scarce resources, cost containment, peer review, and audit, all of which must be considered before a policy is formulated.

The purpose of this around-the-globe sampling from leading interventionists is to compare some perspectives and insights. Through such dialogue and continued research we hope that with time the question of surgical cover will become less polemic. In the meantime all our interventionists agree that to get good PTCA results an operator must have a large case load, that surgical cover is clinically less important than it was, and that for a variety of reasons the development of new centres without on site surgery would be unwelcome.

PETER MILLS

MARTIN T ROTHMAN ERIC J TOPOL
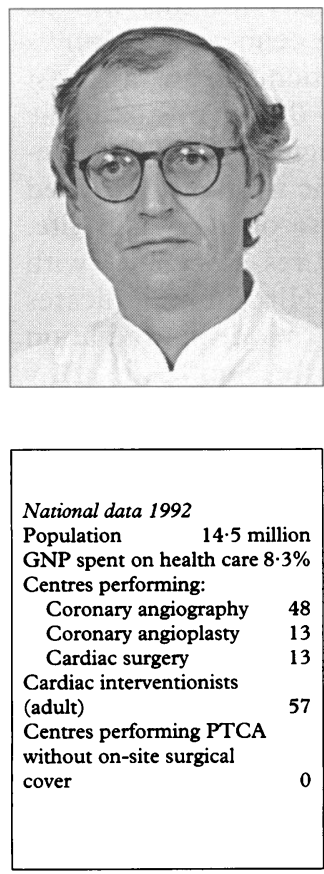

\begin{tabular}{|lr|}
\hline & \\
Data on institution 1992 & \\
Angiograms & 2516 \\
CABGs & 1143 \\
PTCAs & 1382 \\
Other adult coronary & \\
interventions & 209 \\
Coronary interventions in & \\
adults: & \\
Single vessel disease & 843 \\
Multi-vessel disease & 539 \\
Disease in grafts & 62 \\
Stents & 15 \\
Interventionists & 5 \\
\hline
\end{tabular}

\section{The Netherlands}

I think that the question whether PTCA needs on-site surgical cover is much less important than the quality requirements for PTCA. Quality is strongly related to quantity; in fact it is a prerequisite for quality. Large case loads are also required to maintain and improve the skills and expertise of the interventionists, especially with bail-out devices and new techniques.

The present situation in the Netherlands is very good. There are 13 centres covering the Netherlands quite evenly, the mean yearly case load per centre is well over 750 , and the mean yearly case load per interventionist is 175 . In 1992 procedural mortality from PTCA was $0.3 \%$, the acute myocardial infarction rate as a complication of PTCA was $1.8 \%$, and the emergency CABG rate was $1 \cdot 2 \%$. These excellent results reflect the experience of the operators and their teams, the quality of their equipment, and the daily dialogue with the surgeons on indications and strategies before, during, and after the procedure. The importance of on-site consultation with other highly experienced interventionists, cardiac surgeons, and cardiac anaesthetists should not be underestimated.

A continuous service, 365 days a year and 24 hours a day, requires at least three interventionists. To allow these interventionists to perform 175 cases per year, the present average in the Netherlands, requires a hospital PTCA case load of over 500 . If case selection is to be performed to identify high risk patients, about 750 patients would need to be recruited every year.

The guidelines of the Working Group on Interventional Cardiology of the Dutch Society of Cardiology recommend at least 300 cases per centre and 100 cases per operator. No Dutch hospital with catheterisation laboratories without surgical facilities provides 300 candidates for PTCA per year. Because none of these hospitals would meet the Dutch national standards some would have to pool their patients to form a new cardiac surgical centre.

Are there any other reasons for performing PTCA in non-surgical centres? If long waiting lists are an issue, we need to agree on an acceptable waiting time. For semi-acute cases this should be less than a week and for elective cases less than 3 months. If waiting times are longer, opening up one new large (surgical) centre would then be a much wiser decision than allowing PTCA to be performed in non-surgical centres.

In my opinion extending the ability of more hospitals to perform angioplasty in patients with acute infarcts or with other acute syndromes does not justify PTCA without on-site surgical cover. The results of the present thrombolytic strategies are excellent, the number of infarct patients with contraindications to thrombolysis is small, and the level of competence in angioplasty required in such cases is very high. If low risk cases are selected for treatment at smaller cardiac units, such units should not perform PTCA in acute myocardial infarction. In the Netherlands, with the small distances between surgical and angioplasty centres, it would probably not be a good use of resources to allow smaller hospitals to perform PTCA on patients with acute myocardial infarction.

Currently the Dutch Ministry of Health insists on surgical back-up for PTCA, so that PTCA is restricted to surgical centres. I conclude that it would be unwise to uncouple angioplasty from surgery in the Netherlands.

$\underline{\underline{m}}$ . 


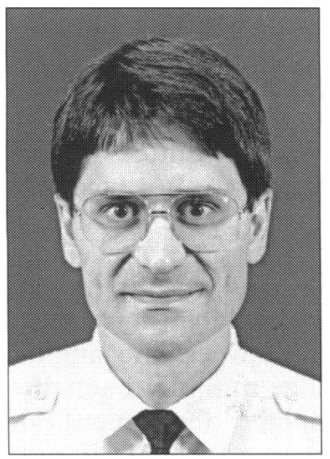

National data 1992 Population

7 million

NP spent on health care $9 \%$

Centres performing: Coronary angiography Coronary angioplasty Cardiac surgery

Cardiac interventionists

(adult)

Centres performing PTCA

without on-site surgical cover

\begin{tabular}{|lr|}
\hline Data on institution 1992 & \\
Angiograms & 1506 \\
CABGs & 326 \\
PTCAs & 605 \\
Other adult coronary & \\
interventions & 69 \\
Coronary interventions in & \\
adults: & \\
$\quad$ Single vessel disease & 513 \\
$\quad$ Multi-vessel disease & 92 \\
$\quad$ Disease in grafts & 50 \\
Stents & 44 \\
Interventionists & 7 \\
&
\end{tabular}

Interventionists

\section{Switzerland}

The expressions "surgical stand-by" or "surgical backup" featured in the glossary of coronary balloon angioplasty even before it became established. The first emergency bypass operation for failed angioplasty was performed on Gruentzig's seventh patient.

Two types of emergency surgery have results that compare well with those of elective surgery. They are emergency surgery for convenience (for practical rather than medical reasons) and emergency surgery for threatened infarction (the most effective application of surgical back-up). The value of emergency surgery for ongoing myocardial infarction is debatable. Its periprocedural infarction rate is by definition $100 \%$ and its mortality is greater than $20 \%$.

The prerequisites for the ideal surgical back-up are exacting, costly, and rarely met. They include an operating theatre immediately available for 24 hours and accessible to patients who require life support measures.

In 1992 all angioplasty centres in Switzerland had onsite cardiac surgery: since then this has changedthough according to the national guidelines surgical stand-by, specifically in-house surgical cover, is mandatory for all coronary angioplasty procedures. Typically, in institutions with a busy interventional and surgical programme, the "open theatre" policy has long been replaced by the "next theatre available" policy. In several centres interventional cardiology and cardiac surgery function independently and deal with casualties as they arise.

The coronary stent is the only new angioplasty device that had a significant impact on the incidence of acute vessel closure and the need for emergency surgery. It has, however, by no means eliminated the possibility of acute vessel closure. A more important reason for reducing emergency surgery rates has been the realisation by interventional cardiologists that patients with a small area of ischaemia may be harmed by surgery. Patients who have small or medium sized myocardial infarctions in hospital and are treated conservatively are unlikely to die and have an excellent functional prognosis. I believe that the use of emergency surgery for failed angioplasty has had a negative impact on the overall death toll.

In countries with abundant surgical facilities surgical stand-by should not be requested for angioplasty procedures. This policy would avoid wasting money on services that are not used. It would also counter the incentive to create new surgical programmes just to do angioplasty without breaking the rules. Such facilities are bound to have low indication thresholds and to divert patients from established high-volume surgical institutions. In countries with waiting lists for coronary angioplasty and surgery, the request for surgical standby for angioplasty procedures should be abandoned too, because a lengthening waiting list results in more deaths than angioplasty without surgical back-up. Proscription of coronary angioplasty without full surgical cover is like banning cars without anti-lock brakes and air bags. It enhances safety but stops the traffic.

Undoubtedly a skilled surgical team ready to take over immediately in the event of a problem in the interventional (or diagnostic) laboratory is a welcome safety net and may save myocardium and even an occasional life, if called upon judiciously. But organising surgical cover should not be allowed to hamper angioplasty activity nor should emergency surgery be overused or the need for it overestimated. It has no role with an ongoing small or medium size infarction where it may be called upon merely to calm the operator. It probably has no role at the other extreme either, when vigorous prolonged, and futile resuscitation efforts are made on a patient on the catheterisation table whose dominant left main stem has been dissected by a guiding catheter.

All in all, surgical back-up has become to coronary angioplasty what a teddy bear is in the bed of a 10 year old-no longer required but nice to have around.

BERNHARD MEIER

University Hospital, Bern

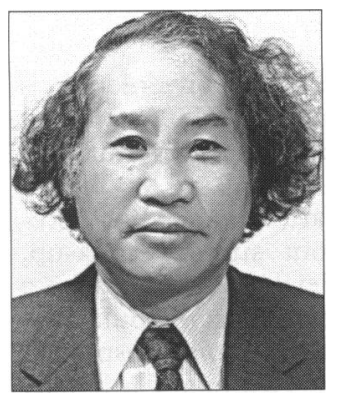

National data 1992

Population 120 million GNP spent on health care Centres performing: Coronary angiography 1000 Coronary angioplasty 500 Cardiac surgery Cardiac interventionists (adult)

Centres performing PTCA

pen-site surgical

cover

480

300

Data on institution 1992 Angiograms

CABGs

CABG

Other adult coronary

interventions

Coronary interventions in

adults:

Single vessel disease

Multi-vessel disease

Disease in grafts

Stents

Interventionists

439

1143

\section{Japan}

Percutaneous transluminal coronary angioplasty has progressed dramatically since its inception 16 years ago. The precision of equipment and instruments, experience and knowledge about the indications and limits of intervention, and systems for detecting and solving problems within the hospital have all advanced to the point that Gruentzig would probably not recognise the protocols we use today for PTCA.

Though an excellent working relationship and close coordination with a surgical team are indispensable to the interventional cardiologist I do not believe that basic procedures done by reasonably experienced practitioners need on-site cardiac surgery. An interventional procedure on an infarct related artery, for example, is unlikely to need surgical stand-by. In general, if acute mortality for PTCA can be held at less than $0.1 \%$, well in line with current practice in Japan, I would not deem that surgical stand-by is necessary. Nonetheless, operators must be qualified, and informed consent must always be obtained for any invasive or interventional procedure; consent should include the possibility of surgery and if the surgical cover is off site this fact should be made explicit in the consent form.

Complex procedures or those that involve atherectomy or scaffolding devices must be done with on-site surgical back-up. Work on left main vessel, for example, should always have surgical support because any damage to the vessel, including dissection, will require surgical intervention. I routinely use three different coronary stents and five different atherectomy devices, and I always have on-site surgical cover for all procedures. Neither the Japanese government nor scientific societies have established guidelines on surgical stand-by. This is left to the interventionist and the institution. As interventional cardiology progresses, formal guidelines may develop.

In conclusion, interventional cardiologists should always maintain close contact with surgeons regarding their protocol and practice. Though on-site surgery may not be available to all, its availability should certainly be regarded as a limiting factor if new interventions and expanded indications are being considered. The more complex and difficult the case, whatever of the skill level of the practitioner, the greater the weight that should be given to surgical support.

MASAKIYO NOBUYOSHI Department of Cardiology, Kokura Memorial Hospital, Kitakyushu 


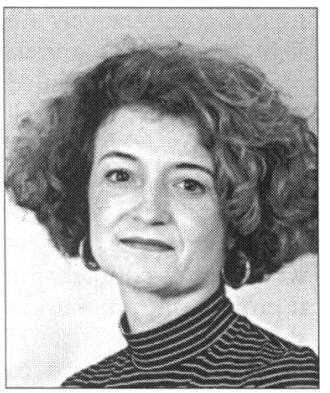

National data 1992

Population 55 million GNP spent on health care $9 \%$ Centres performing:

Coronary angiography $\quad 175$

Coronary angioplasty 139

Cardiac surgery

Cardiac interventionists

(adult)

Centres performing PTCA

without on-site surgical

cover

\begin{tabular}{|lr|}
\hline Data on institution 1992 & \\
Angiograms & 2347 \\
CABGs & 220 \\
PTCAs & 1080 \\
Other adult coronary & \\
interventions & 65 \\
$\begin{array}{l}\text { Coronary interventions in } \\
\text { adults: }\end{array}$ & \\
$\begin{array}{l}\text { Single vessel } \\
\text { disease }\end{array}$ & $516(47 \cdot 7 \%)$ \\
$\begin{array}{l}\text { Multi-vessel } \\
\text { disease }\end{array}$ & $564(52 \cdot 2 \%)$ \\
$\begin{array}{l}\text { Disease in grafts } \\
\text { Stents }\end{array}$ & $27(2 \cdot 5 \%)$ \\
Interventionists & $50(4 \cdot 6 \%)$ \\
& \\
\hline
\end{tabular}

\section{France}

PTCA without on-site surgery is acceptable if the catheterisation laboratory is not too far from the operating theatre and if emergency surgery is well organised. When all the operating theatres are occupied PTCA departments with on-site surgery face the same problem as those with none. At the Centre Cardiologique de Nord the patient is told that emergency surgery may be necessary and how it is carried out. Large series of published PTCA results serve as reference data. We enter the results for each team on a computerised database and compare them with the results of others. Then either their indications or their technique can be modified if the results fall short of those reported by others.

The availability of perfusion balloons and stents has dramatically reduced the rate of emergency surgery (less than $0.5 \%$ in my institute), moreover they often allow artery patency to be re-established in patients who have to have surgery, which provides a calmer situation. Some complications, however, cannot be resolved by perfusion balloons and stents alone, and for these rare patients strict organisation of emergency surgery is essential. Occlusive dissection of the left main coronary artery is the worst situation. It is extremely rare $(<1 \%)$ but very serious even with on-site surgery because the outcome in a patient operated on during cardiac arrest is extremely poor. I think that everything should be done to restore the patency of the left main coronary artery by angioplasty-including balloons, stents, or flow support cathetersbefore the patient has surgery with counterpulsation or cardiopulmonary support.
In 1992 I was part of a team that set up guidelines for coronary angiography and angioplasty at the request of the ethics committee of the French Society of Cardiology. We recommended that for centres with on-site surgery there should be less than 1 hour of travelling between the decision to operate and arrival in the operating theatre. The guidelines recommend a signed agreement between the angioplasty and surgery teams. The minimum activity required from a good angioplasty department (at least 300 procedures/year) is of equal, if not greater importance, and there must also be guaranteed 24 hours a day availability.

At the Centre Cardiologique du Nord we conform completely with these recommendations: the surgical team is not more than 15 minutes $(3 \mathrm{~km})$ away and each day before the angioplasty procedures start we ask both surgeons and the doctors on the mobile intensive care unit if we can go on stand-by, even for patients with total chronic occlusion. The only exception is when we perform emergency PTCA for acute myocardial infarction, which can happen at any time of the day or the night, because the culprit artery is already occluded.

At the Centre Cardiologique du Nord, we believe that in experienced hands coronary angiography is now such a safe procedure that it does not require surgical stand-by.

MARIE-CLAUDE MORICE Centre Cardiologique du Nord, Saint-Denis, Paris

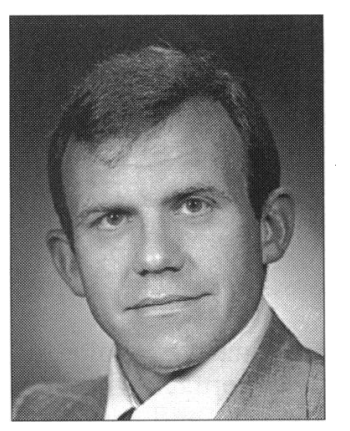

National data 1992 Population 250 million GNP spent on health

GNP spent on health $10-11 \%$

Centres performing

Coronary angiography 1600 Coronary angioplasty 1100 Cardiac surgery Cardiac interventionists (adult)

Centres performing

PTCA without on-site surgical cover

$-6000$

Data on institution 1992 Angiograms

CABGs

PTCA

Other adult coronary

interventions

Coronary interventions in

adults:

Single vessel disease

Multi-vessel disease

Disease in grafts

Stents

Interventionists

\section{United States}

16 years after the introduction of PTCA the outcome of individual procedures continues to be unpredictable. Lesion characteristics associated with increased risk of closure have been identified in population studies, but for a specific patient the risk is hard to predict. Though they are infrequent, complications are often unpredictable and they can occur with experienced operators treating ideal lesions with optimal equipment. Acute closure results in adverse outcome with increased rates of infarction, emergency surgery (coronary artery bypass grafting (CABG)), and even death. In addition, the outcome of surgery carried out under these conditions is poorer than that of elective CABG.

Intracoronary stents have improved outcome after dissection and abrupt closure; stents, however, do not always solve the problem. In the Cook registry of stents placed for acute or threatened closure, $5-7 \%$ of patients required emergency CABG. Local arterial factors or insufficient guiding catheter support can prevent the delivery of stents.

Performance of interventional procedures without on-site surgery usually arises from the cardiologist or institution wishing to offer a partial "full service bank" - that is, without cardiac surgery. Given that the surgical facility must be relatively close in case urgent transport is required, the need to be a partial full service bank leads to considerable redundancy in the system because interventional procedures are usually also available at the site where surgery is performed. Patient convenience is not a persuasive argument for PTCA without on-site surgery. By definition surgery must be close at hand.

When a patient with infarction and shock presents to a hospital without surgical back-up, should the laboratory undertake what may be a lifesaving procedure or alternatively should that patient be triaged and sent by urgent transport to $\delta$ the nearby centre with interventional cardiology and on-site surgical coverage? While this is a real 을 concern, in general, the sicker the patient, the $D$ greater the breadth of services and clinical experience required to optimise care. It makes more sense to urgently transport the patient to the o most experienced centre; or, perhaps ideally, N ambulance services could preferentially deliver patients to these centres.

In the United States there is growing concern about the mounting cost of medical care. Invasive cardiological procedures are a substan- $\stackrel{\Phi}{\rightarrow}$ tial part of those costs. What is probably in the best interest of both patient care and health care 0 delivery systems is not more "partial full service $\mathbb{D}$ banks" without on site surgery but more central- $\frac{\rho}{\mathbb{D}}$ isation and development of regional centres that $\cong$ provide easy and prompt access to interventional cardiology and surgical services with the care 8 delivered by true experts and continuing quality assurance programmes to document and study outcomes.

DAVID R HOLMES, JR Mayo Clinic, Rochester, Minnesota

\section{. . .

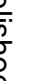

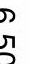

\section{.}

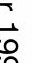

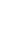

